\title{
Sometimes Pooled Testimation in the Inverse Gaussian Model for Measure of Dispersion
}

\author{
G. Prakash $^{1^{*}}$ and D. C. Singh ${ }^{2}$ \\ ${ }^{1}$ Evaluation Division, State Planning Institution, Lucknow, U. P., India \\ ${ }^{2}$ Department of Statistics, Harish Chandra P. G. College, Varanasi, U. P., India
}

\begin{abstract}
This paper suggests sometimes pool estimators for the measure of dispersion in the inverse Gaussian distribution and their properties are studied in terms of the relative bias and relative efficiency under two different loss functions.

Keywords: Sometimes pool estimator; Level of significance; Relative bias; Relative efficiency; Effective interval.

C) 2010 JSR Publications. ISSN: 2070-0237 (Print); 2070-0245 (Online). All rights reserved.

DOI: 10.3329 /jsr.v2i1.2809

J. Sci. Res. 2 (1), 77-86 (2010)
\end{abstract}

\section{Introduction}

The inverse Gaussian distribution has useful applications in a wide variety of fields such as Biology, Economics and Medicine [1-3]. It plays an important role in reliability theory and life testing problems. Let $x$ be the inverse Gaussian variate with the parameters $\mu$ (measure of location) and $\sigma$ (measure of dispersion), having probability density function

$$
f(x)=\sqrt{\left(2 \pi \sigma x^{3}\right)^{-1}} \exp \left(-\frac{(x-\mu)^{2}}{2 \sigma \mu^{2} x}\right) ; x>0, \sigma, \mu>0 \text {. }
$$

Here, $\sigma^{-1}$ stands as the shape parameter. Let $x_{i 1}, x_{i 2}, x_{i 3}, \ldots, x_{i n_{i}} ; i=1,2$ be two independent random samples of size $n_{1}$ and $n_{2}$ drawn from two inverse Gaussian distributions. The maximum likelihood estimates of $\mu_{i}$ and $\sigma_{i}^{-1} ; i=1,2$ are given as

$$
\hat{\mu}_{i}=\frac{1}{n_{i}} \sum_{j=1}^{n_{i}} x_{i j}=\bar{x}_{i}
$$

and

\footnotetext{
*Corresponding author: ggyanji@yahoo.com
} 


$$
\hat{\sigma}_{i}^{-1}=\frac{n_{i}}{v_{i}} ; v_{i}=\sum_{j=1}^{n_{i}}\left(\frac{1}{x_{i j}}-\frac{1}{\bar{x}_{i}}\right) \text {. }
$$

Also, $\bar{x}_{i}$ and $\hat{\sigma}_{i}=v_{i}\left(n_{i}-1\right)^{-1}$ are the unbiased estimates of $\mu_{i}$ and $\sigma_{\mathrm{i}} ; \mathrm{i}=1,2$, respectively. The pool unbiased estimator for $\sigma$ is given as

$$
\bar{\sigma}=\frac{v_{1}+v_{2}}{n_{1}+n_{2}-2} .
$$

In many real life situations the overestimation and underestimation are not of equal consequences. For such situations a symmetric loss function like as a square error loss (SELF) function is not appropriate [4-6]. A useful asymmetric loss function was introduced by [4], named as the LINEX loss function. The invariant version of the LINEX loss function (ILLF) [7] for the parameter $\theta$ is given as

$$
L(\Delta)=e^{a \Delta}-a \Delta-1 ; a \neq 0 \text { and } \Delta=(\hat{\theta}-\theta) \theta^{-1} .
$$

The LINEX loss function rises approximately exponentially on one side of zero and approximately linearly on the other side. Here, $\hat{\theta}$ is an estimate of the parameter $\theta$. The sign and magnitude of ' $a$ ' represent the direction and degree of asymmetry respectively. The positive (negative) value of ' $a$ ' is used when overestimation is more (less) serious than underestimation. For ' $a$ ' close to zero, the LINEX loss is approximately squared error and therefore almost symmetric. Recently, some shrinkage testimators for the inverse dispersion of the inverse Gaussian distribution under the ILLF has been studied elsewhere [7].

Han and Bancroft [8] have studied the sometimes pool estimator for the mean of a Normal distribution. They have considered the situation when two independent random samples are available from two Normal distributions with means $\mu_{1}$ and $\mu_{2}$ and the common variance. The problem of pooling in different situations has also been considered by other workers [9-12]. Rai [13] has estimated the mean life of Exponential distribution. Sometimes pool estimator for shape parameter of the Pareto distribution under the SELF has been proposed by [14].

In the present article, we have studied the performances of the sometimes pool estimators for $\sigma$ under the SELF and ILLF.

\section{The Proposed Class of Estimators}

We consider a class of estimators for $\sigma$ of the model (1) as

$$
Y=C \hat{\sigma}_{1} ; C \in R^{+}
$$

The risk of $Y$ under the SELF and ILLF are obtained respectively as

$$
R_{(\mathrm{S})}(Y)=\sigma_{1}^{2}\left\{C^{2} \frac{\varphi_{1}+1}{\varphi_{1}}-2 C+1\right\},
$$


and

$$
R_{(L)}(Y)=e^{-a}\left(1-\frac{a C}{\varphi_{1}}\right)^{-\varphi_{1}}+a(1-C)-1 ; \varphi_{1}=\frac{n_{1}-1}{2}
$$

The suffix $S$ and $L$ stand respectively for the risk under the SELF and ILLF criteria. The values of $C$ which minimize the risks $R_{(S)}(Y)$ and $R_{(L)}(Y)$ respectively are given as

$$
C_{1}=\frac{\varphi_{1}}{\varphi_{1}+1} \text { and } C_{2}=\frac{\varphi_{1}}{a}\left\{1-\exp \left(-\frac{a}{\varphi_{1}+1}\right)\right\} \text {. }
$$

The minimum risk estimators of $\sigma$ in the class $Y$ with their respective risks are given as

\begin{tabular}{ll}
\hline Estimator & Risks \\
\hline$Y_{1}=C_{1} \hat{\sigma}_{1}$ & $R_{(S)}\left(Y_{1}\right)=\frac{\sigma_{1}^{2}}{\varphi_{1}+1}$ \\
$R_{(L)}\left(Y_{1}\right)$ & $=e^{-a}\left(\frac{\varphi_{1}+1}{\varphi_{1}+1-a}\right)^{\varphi_{1}}-\left(\frac{\varphi_{1}+1-a}{\varphi_{1}+1}\right)$ \\
& $R_{(S)}\left(Y_{2}\right)=\sigma_{1}^{2}\left\{C_{2}^{2} \frac{\varphi_{1}+1}{\varphi_{1}}-2 C_{2}+1\right\}$ \\
$Y_{2}=C_{2} \hat{\sigma}_{1} \quad$ & $R_{(L)}\left(Y_{2}\right)=\left(\varphi_{1}+1\right)\left(\exp \left(-\frac{a}{\varphi_{1}+1}\right)-1\right)+a$
\end{tabular}

\section{The Proposed Pool Class of Estimators}

We consider a class of the pooled estimator for $\sigma$ of the distribution (1) as

$$
\hat{S}=l \bar{\sigma} ; l \in R^{+}
$$

The expressions of the risks for $\hat{S}$ under the SELF and ILLF, respectively are given as

$$
R_{(S)}(\hat{S})=\sigma_{1}^{2}\left\{l^{2} \frac{\varphi_{1}+\varphi_{2}+1}{\varphi_{1}+\varphi_{2}}-2 l+1\right\} ; \varphi_{2}=\frac{n_{2}-1}{2}
$$

and 


$$
R_{(L)}(\hat{S})=e^{-a}\left(1-\frac{a l}{\varphi_{1}+\varphi_{2}}\right)^{-\varphi_{1}-\varphi_{2}}+a(1-l)-1
$$

The values of $l$ for which the risks $R_{(S)}(\hat{S})$ and $R_{(L)}(\hat{S})$ are minimum, are given respectively as

$$
l_{1}=\frac{\varphi_{1}+\varphi_{2}}{\varphi_{1}+\varphi_{2}+1} \text { and } l_{2}=\frac{\varphi_{1}+\varphi_{2}}{a}\left\{1-\exp \left(-\frac{a}{\varphi_{1}+\varphi_{2}+1}\right)\right\} \text {. }
$$

The improved classes of pooled estimator for $\sigma$ with their respective risks are given as

$\frac{\text { Estimator }}{\hat{S}_{1}=l_{1} \bar{\sigma}} \quad R_{(S)}\left(\hat{S}_{1}\right)=\frac{\sigma_{1}^{2}}{\varphi_{1}+\varphi_{2}+1}$
$\hat{S}_{2}=l_{2} \bar{\sigma} \quad R_{(L)}\left(\hat{S}_{2}\right)=\left(\varphi_{1}+\varphi_{2}+1\right)\left(\exp \left(-\frac{a}{\varphi_{1}+\varphi_{2}+1}\right)-1\right)+a$

\section{The Proposed Sometimes Pool Estimators and their Properties}

Our interest is to estimate the parameter $\sigma_{l}$ when it is suspect but not known for certain that $\sigma_{1}=\sigma_{2}$. Before pooling the two sample estimates for the estimation of the parameter $\sigma$, the test of hypothesis $H_{0}: \sigma_{1}=\sigma_{2}$ may be performed at some pre-assigned level of significance $\alpha$. The test statistic for $H_{0}$ is given as

$$
F=\frac{\left(n_{2}-1\right) v_{1}}{\left(n_{1}-1\right) v_{1}} \sim F_{\left(n_{1}-1\right),\left(n_{2}-1\right)} .
$$

The proposed sometimes pooled estimator is given as

$$
\hat{\sigma}_{S T_{i}}=\left\{\begin{array}{ll}
l_{i} \bar{\sigma} & \text { if } f_{1} \leq F \leq f_{2} \\
C_{i} \hat{\sigma}_{1} & \text { else }
\end{array},\right.
$$

where $i=1,2$ and $f_{1}, f_{2}$ are the lower and upper $100 \alpha / 2 \%$ points of the $F$ distribution with $\left(n_{1}-1\right)$ and $\left(n_{2}-1\right)$ degrees of freedom. The hypothesis $H_{0}$ is rejected when $F \leq f_{1}$ or $F \geq f_{2}$ and $P_{H_{0}}\left[F \leq f_{1}\right.$ or $\left.F \geq f_{2}\right]=\alpha$. Thus if $\alpha=0, \hat{\sigma}_{S T_{i}}=l_{i} \bar{\sigma}$ and it is always pool 
estimator. If $\alpha=1, \hat{\sigma}_{S T_{i}}=C_{i} \hat{\sigma}_{1}$ and it is never pool estimator. Otherwise it is sometimes pool estimator.

The relative biases for the estimators $\hat{\sigma}_{S T_{i}} ; i=1,2$ are obtained as

$$
\begin{aligned}
R B\left(\hat{\sigma}_{S T_{i}}\right) & =\frac{E\left(\hat{\sigma}_{S T_{i}}\right)}{\sigma_{1}}-1 \\
& =G\left\{F_{1}, F_{2},\left(\Delta_{i 1}-\Delta_{i 0}\right), 0,0\right\}+C_{i}-1,
\end{aligned}
$$

where $\Delta_{i 1}=\frac{l_{i}\left(\delta+Z_{2}\right)}{\delta\left(\varphi_{1}+\varphi_{2}\right)}, \Delta_{i 0}=\frac{C_{i}}{\varphi_{1}}, F_{1}=\left(\frac{n_{2}-1}{n_{1}-1}\right) \frac{\delta}{f_{2}}, \delta=\frac{\sigma_{1}}{\sigma_{2}}, F_{2}=\left(\frac{n_{2}-1}{n_{1}-1}\right) \frac{\delta}{f_{1}}$,

$$
G\left\{F_{1}, F_{2}, w_{1}, w_{2}, r\right\}=\frac{\Gamma\left(\varphi_{1}+\varphi_{2}+1+r\right)}{\Gamma \varphi_{1} \Gamma \varphi_{2}} \int_{F_{1}}^{F_{2}} \frac{w_{1} Z_{2}^{\varphi_{2}-1}}{\left(1+Z_{2}-w_{2}\right)^{\varphi_{1}+\varphi_{2}+1+r}} d Z_{2}
$$

and $w_{1}$ and $w_{2}$ are functions of $Z_{2}$.

The expressions of the risks under the SELF and ILLF for $\hat{\sigma}_{S T_{i}} ; i=1,2$ are obtained as

$$
\begin{aligned}
R_{(S)}\left(\hat{\sigma}_{S T_{i}}\right) & =\sigma_{1}^{2}\left\{G\left\{F_{1}, F_{2},\left(\Delta_{i 1}^{2}-\Delta_{i 0}^{2}\right), 0,1\right\}\right. \\
& \left.-2 G\left\{F_{1}, F_{2},\left(\Delta_{i 1}-\Delta_{i 0}\right), 0,0\right\}+1-C_{i}\right\}
\end{aligned}
$$

and

$$
\begin{aligned}
R_{(L)}\left(\hat{\sigma}_{S T_{i}}\right)= & e^{-a}\left\{G\left\{F_{1}, F_{2}, 1, a\left(\Delta_{i 1}-\Delta_{i 0}\right),-1\right\}\right. \\
& \left.+G\left\{0, \infty, 1, a \Delta_{i 0},-1\right\}\right\}+a\left(1-C_{i}\right)-1 \\
& -a G\left\{F_{1}, F_{2},\left(\Delta_{i 1}-\Delta_{i 0}\right), 0,0\right\} ; i=1,2 .
\end{aligned}
$$

The relative efficiencies for the pooled estimator $\hat{\sigma}_{S T_{1}}$ with respect to $Y_{1}$ and $\hat{\sigma}_{S T_{2}}$ with respect to $Y_{2}$ under the SELF and ILLF criteria are defined as

and

$$
R E_{(S)}\left(\hat{\sigma}_{S T_{i}}, Y_{i}\right)=R_{(S)}\left(Y_{i}\right) / R_{(S)}\left(\hat{\sigma}_{S T_{i}}\right)
$$

$$
R E_{(L)}\left(\hat{\sigma}_{S T_{i}}, Y_{i}\right)=R_{(L)}\left(Y_{i}\right) / R_{(L)}\left(\hat{\sigma}_{S T_{i}}\right) \text {. }
$$

The expressions of the relative biases and relative efficiencies are the functions of $n_{1}$, $n_{2}, \delta, a$ and $\alpha$. For the selected set of values of $\left(n_{1}, n_{2}\right)=05,08,10 ; \delta=0.60(0.20) 1.80$; $a= \pm 0.25, \pm 0.50$ and $\alpha=0.01,0.05,0.10,0.15$ the relative biases and relative efficiencies have been calculated. The 16-point Gauss-Legendre quardature formula is used to solve the integrations involved in relative biases and relative efficiencies. The relative biases are not presented here and the relative efficiencies have been presented in the Tables 1 to 4 for $\alpha=0.01$ and 0.05 . 
Table 1. Relative efficiency between $\hat{\sigma}_{S T_{1}}$ and $Y_{1}$ under SELF.

\begin{tabular}{|c|c|c|c|c|c|c|c|c|}
\hline \multicolumn{2}{|c|}{$\alpha=0.01$} & \multicolumn{7}{|c|}{$\delta$} \\
\hline$n_{1}$ & $n_{2}$ & 0.60 & 0.80 & 1.00 & 1.20 & 1.40 & 1.60 & 1.80 \\
\hline \multirow{3}{*}{05} & 05 & 1.5406 & 2.1106 & 2.7387 & 2.4039 & 2.2887 & 2.1675 & 2.0579 \\
\hline & 08 & 1.4563 & 2.4582 & 3.4010 & 2.9121 & 2.6405 & 2.3816 & 2.1672 \\
\hline & 10 & 1.4395 & 2.6305 & 3.7701 & 3.1776 & 2.7969 & 2.4526 & 2.1826 \\
\hline \multirow{3}{*}{08} & 05 & 1.4683 & 1.9306 & 2.4863 & 2.1903 & 2.0973 & 1.9972 & 1.9059 \\
\hline & 08 & 1.3558 & 2.1473 & 2.9177 & 2.5092 & 2.2952 & 2.0899 & 1.9199 \\
\hline & 10 & 1.2717 & 2.3718 & 3.4216 & 2.8622 & 2.4941 & 2.1754 & 1.9335 \\
\hline \multirow{3}{*}{10} & 05 & 1.4421 & 1.8927 & 2.4432 & 2.1527 & 2.0603 & 1.9615 & 1.8072 \\
\hline & 08 & 1.3378 & 1.9756 & 2.6143 & 2.2598 & 2.1014 & 1.9869 & 1.8154 \\
\hline & 10 & 1.2376 & 2.2284 & 3.1354 & 2.6236 & 2.3130 & 2.0419 & 1.8319 \\
\hline \multicolumn{9}{|c|}{$\alpha=0.05$} \\
\hline \multirow{3}{*}{05} & 05 & 1.4924 & 1.9694 & 2.5446 & 2.2366 & 2.1335 & 2.0255 & 1.9296 \\
\hline & 08 & 1.4482 & 2.2873 & 3.0479 & 2.5793 & 2.3458 & 2.1401 & 1.9790 \\
\hline & 10 & 1.4212 & 2.4401 & 3.3005 & 2.7331 & 2.4267 & 2.1756 & 1.9893 \\
\hline \multirow{3}{*}{08} & 05 & 1.4266 & 1.7779 & 2.2942 & 2.0447 & 1.9778 & 1.8977 & 1.8114 \\
\hline & 08 & 1.3226 & 1.9333 & 2.5948 & 2.2581 & 2.1007 & 1.9439 & 1.8121 \\
\hline & 10 & 1.2472 & 2.0786 & 2.8991 & 2.4678 & 2.2177 & 1.9921 & 1.8166 \\
\hline \multirow{3}{*}{10} & 05 & 1.4124 & 1.7403 & 2.2506 & 2.0132 & 1.9513 & 1.8231 & 1.7171 \\
\hline & 08 & 1.3163 & 1.7975 & 2.3847 & 2.0981 & 1.9770 & 1.8486 & 1.7273 \\
\hline & 10 & 1.2257 & 1.9396 & 2.7256 & 2.3484 & 2.1186 & 1.9038 & 1.7363 \\
\hline
\end{tabular}

Table 2. Relative efficiency between $\hat{\sigma}_{S_{T_{2}}}$ and $Y_{2}$ under SELF for $\alpha=0.01$.

\begin{tabular}{ccccccccc}
\hline \multicolumn{2}{c}{$a=-0.50$} & \multicolumn{7}{c}{$\delta$} \\
\hline$n_{1}$ & $n_{2}$ & 0.60 & 0.80 & 1.00 & 1.20 & 1.40 & 1.60 & 1.80 \\
\hline \multirow{4}{*}{05} & 05 & 1.7843 & 2.2528 & 2.8015 & 2.4094 & 2.2705 & 2.1382 & 2.0233 \\
& 08 & 1.7431 & 2.7441 & 3.6105 & 3.0089 & 2.6881 & 2.4029 & 2.1740 \\
& 10 & 1.6963 & 3.0198 & 4.1381 & 3.3815 & 2.9162 & 2.5223 & 2.2233 \\
& 05 & 1.5696 & 1.8743 & 2.3225 & 2.0231 & 1.9370 & 1.8521 & 1.7771 \\
\multirow{2}{*}{10} & 08 & 1.4889 & 2.1963 & 2.8413 & 2.3841 & 2.1610 & 1.9630 & 1.8036 \\
& 10 & 1.4164 & 2.3859 & 3.1923 & 2.6053 & 2.2725 & 1.9969 & 1.8177 \\
\hline
\end{tabular}


Table 2 (continued)

\begin{tabular}{|c|c|c|c|c|c|c|c|c|}
\hline \multicolumn{9}{|c|}{$a=-0.25$} \\
\hline \multirow{3}{*}{05} & 05 & 1.6618 & 2.1804 & 2.7664 & 2.4026 & 2.2754 & 2.1489 & 2.0370 \\
\hline & 08 & 1.5903 & 2.6249 & 3.5512 & 2.9945 & 2.6877 & 2.4074 & 2.1800 \\
\hline & 10 & 1.5383 & 2.8687 & 4.0485 & 3.3491 & 2.9018 & 2.5149 & 2.2191 \\
\hline \multirow{3}{*}{10} & 05 & 1.5089 & 1.8400 & 2.3087 & 2.0257 & 1.9480 & 1.8680 & 1.7958 \\
\hline & 08 & 1.4030 & 2.1359 & 2.8219 & 2.3931 & 2.1801 & 1.9853 & 1.8264 \\
\hline & 10 & 1.3243 & 2.3117 & 3.1732 & 2.6198 & 2.2951 & 2.0199 & 1.8292 \\
\hline \multicolumn{9}{|c|}{$a=0.25$} \\
\hline \multirow{3}{*}{05} & 05 & 1.4203 & 2.0355 & 2.7053 & 2.4031 & 2.3022 & 2.1878 & 2.0815 \\
\hline & 08 & 1.3084 & 2.3971 & 3.4532 & 2.9896 & 2.7099 & 2.4360 & 2.2085 \\
\hline & 10 & 1.2554 & 2.5894 & 3.8974 & 3.3100 & 2.8944 & 2.5168 & 2.2237 \\
\hline \multirow{3}{*}{10} & 05 & 1.3879 & 1.7726 & 2.2855 & 2.0372 & 1.9779 & 1.9082 & 1.8420 \\
\hline & 08 & 1.2385 & 2.0187 & 2.7920 & 2.4235 & 2.2319 & 2.0429 & 1.8540 \\
\hline & 10 & 1.1504 & 2.1694 & 3.1498 & 2.6673 & 2.3576 & 2.0805 & 1.8644 \\
\hline \multicolumn{9}{|c|}{$a=0.50$} \\
\hline \multirow{3}{*}{05} & 05 & 1.3302 & 1.9642 & 2.6814 & 2.4129 & 2.3265 & 2.2184 & 2.1146 \\
\hline & 08 & 1.1799 & 2.2908 & 3.4176 & 3.0022 & 2.7348 & 2.4618 & 2.2319 \\
\hline & 10 & 1.1300 & 2.4635 & 3.8394 & 3.3056 & 2.9024 & 2.5264 & 2.2325 \\
\hline \multirow{3}{*}{10} & 05 & 1.3077 & 1.7398 & 2.2767 & 2.0468 & 1.9974 & 1.9333 & 1.8702 \\
\hline & 08 & 1.1603 & 1.9625 & 2.7822 & 2.4459 & 2.2657 & 2.0793 & 1.9197 \\
\hline & 10 & 1.0689 & 2.1021 & 3.1472 & 2.7023 & 2.3994 & 2.1196 & 1.9910 \\
\hline
\end{tabular}

Table 3. Relative efficiency between $\hat{\sigma}_{S T_{1}}$ and $Y_{1}$ under ILLF for $\alpha=0.01$.

\begin{tabular}{ccccccccc}
\hline \multicolumn{6}{c}{$a=-0.50$} & \multicolumn{7}{c}{$\delta$} \\
\hline$n_{1}$ & $n_{2}$ & 0.60 & 0.80 & 1.00 & 1.20 & 1.40 & 1.60 & 1.80 \\
\hline \multirow{2}{*}{05} & 05 & 1.3799 & 1.9940 & 2.7103 & 2.3035 & 2.2241 & 2.1205 & 2.0190 \\
& 08 & 1.2934 & 2.3075 & 3.3753 & 2.8072 & 2.5730 & 2.3237 & 2.1086 \\
& 10 & 1.2572 & 2.4599 & 3.7182 & 3.0405 & 2.6992 & 2.3645 & 2.0947 \\
& 05 & 1.3317 & 1.8079 & 2.4141 & 2.0501 & 1.9887 & 1.9083 & 1.8294 \\
10 & 08 & 1.2364 & 1.8978 & 2.6097 & 2.1720 & 2.0387 & 1.9644 & 1.7661 \\
& 10 & 1.1312 & 2.1173 & 3.1418 & 2.5496 & 2.2669 & 2.0002 & 1.7875 \\
\hline \multirow{2}{*}{$a=-0.25$} & & & & & & & \\
\hline \multirow{2}{*}{05} & 05 & 1.4500 & 2.0422 & 2.7135 & 2.2743 & 2.1796 & 2.0705 & 1.9683 \\
& 08 & 1.3588 & 2.3689 & 3.3763 & 2.7616 & 2.5169 & 2.2713 & 2.0639 \\
& 10 & 1.3151 & 2.5291 & 3.7263 & 3.0026 & 2.6536 & 2.3257 & 2.0650 \\
\multirow{2}{*}{10} & 05 & 1.3790 & 1.8422 & 2.4205 & 2.0323 & 1.9578 & 1.8708 & 1.7891 \\
& 08 & 1.2800 & 1.9281 & 2.6121 & 2.1420 & 2.0007 & 1.8861 & 1.7306 \\
& 10 & 1.1769 & 2.1623 & 3.1626 & 2.4996 & 2.2124 & 1.9524 & 1.7482 \\
\hline
\end{tabular}


Table 3 (continued)

\begin{tabular}{ccccccccc}
\hline \multicolumn{1}{c}{$a=0.25$} & & & & & & & \\
\hline & 05 & 1.6195 & 2.1545 & 2.7380 & 2.2382 & 2.1187 & 2.0013 & 1.8985 \\
& 08 & 1.5262 & 2.5234 & 3.7011 & 2.7075 & 2.4441 & 2.2038 & 2.0083 \\
& 10 & 1.4683 & 2.7087 & 3.7829 & 2.9645 & 2.5998 & 2.2813 & 2.0345 \\
10 & 05 & 1.4915 & 1.9201 & 2.4405 & 2.0055 & 1.9081 & 1.8107 & 1.7250 \\
& 08 & 1.3823 & 1.9997 & 2.6217 & 2.0991 & 1.9442 & 1.8792 & 1.6781 \\
\multirow{2}{*}{$a=0$} & 0.50 & 1.2881 & 2.2697 & 3.1716 & 2.4263 & 2.1317 & 1.8829 & 1.6925 \\
\hline \multirow{2}{*}{05} & 05 & 1.7166 & 2.2149 & 2.7559 & 2.2286 & 2.0992 & 1.9788 & 1.8760 \\
& 08 & 1.6274 & 2.6120 & 3.8272 & 2.6942 & 2.4228 & 2.1844 & 1.9935 \\
& 10 & 1.5633 & 2.8147 & 3.8252 & 2.9593 & 2.5871 & 2.2718 & 2.0304 \\
& 05 & 1.5558 & 1.9622 & 2.4530 & 1.9961 & 1.8890 & 1.7875 & 1.7005 \\
10 & 08 & 1.4405 & 2.0399 & 2.6374 & 2.0850 & 1.9242 & 1.8179 & 1.6597 \\
& 10 & 1.3534 & 2.3306 & 3.2197 & 2.4008 & 2.1033 & 1.8590 & 1.6741 \\
\hline
\end{tabular}

Table 4. Relative efficiency between $\hat{\sigma}_{S T_{2}}$ and $Y_{2}$ under ILLF for $\alpha=0.01$.

\begin{tabular}{|c|c|c|c|c|c|c|c|c|}
\hline \multicolumn{5}{|c|}{$a=-0.50$} & \multicolumn{3}{|l|}{$\delta$} & \multirow[b]{2}{*}{1.80} \\
\hline$n_{1}$ & $n_{2}$ & 0.60 & 0.80 & 1.00 & 1.20 & 1.40 & 1.60 & \\
\hline \multirow{3}{*}{05} & 05 & 1.6066 & 2.1385 & 2.7699 & 2.4378 & 2.3240 & 2.2003 & 2.0865 \\
\hline & 08 & 1.5492 & 2.5666 & 3.5634 & 3.0552 & 2.7567 & 2.4670 & 2.2262 \\
\hline & 10 & 1.5103 & 2.7989 & 4.0536 & 3.4129 & 2.9685 & 2.5651 & 2.2513 \\
\hline \multirow{3}{*}{10} & 05 & 1.4730 & 1.8150 & 2.3065 & 2.0414 & 1.9729 & 1.8967 & 1.8255 \\
\hline & 08 & 1.3671 & 2.1024 & 2.8365 & 2.4358 & 2.2284 & 2.0290 & 1.8627 \\
\hline & 10 & 1.2947 & 2.2694 & 3.1904 & 2.6717 & 2.3479 & 2.0621 & 1.8797 \\
\hline \multicolumn{9}{|c|}{$a=-0.25$} \\
\hline \multirow{3}{*}{05} & 05 & 1.5747 & 2.1235 & 2.7522 & 2.4189 & 2.3047 & 2.1824 & 2.0710 \\
\hline & 08 & 1.4984 & 2.5385 & 3.5312 & 3.0214 & 2.7253 & 2.4422 & 2.2085 \\
\hline & 10 & 1.4518 & 2.7625 & 4.0103 & 3.3681 & 2.9306 & 2.5384 & 2.2347 \\
\hline \multirow{3}{*}{10} & 05 & 1.4610 & 1.8106 & 2.3014 & 2.0358 & 1.9672 & 1.8916 & 1.8214 \\
\hline & 08 & 1.3438 & 2.0897 & 2.8211 & 2.4210 & 2.2160 & 2.0204 & 1.8578 \\
\hline & 10 & 1.2657 & 2.2549 & 3.1751 & 2.6562 & 2.3356 & 2.0548 & 1.8671 \\
\hline
\end{tabular}


Table 4 (continued)

\begin{tabular}{|c|c|c|c|c|c|c|c|c|}
\hline \multicolumn{9}{|c|}{$a=0.25$} \\
\hline \multirow{3}{*}{05} & 05 & 1.5035 & 2.0915 & 2.7158 & 2.3814 & 2.2669 & 2.1481 & 2.0416 \\
\hline & 08 & 1.3901 & 2.4780 & 3.4648 & 2.9541 & 2.6645 & 2.3948 & 2.1748 \\
\hline & 10 & 1.3294 & 2.6863 & 3.9258 & 3.2831 & 2.8596 & 2.4889 & 2.2047 \\
\hline \multirow{3}{*}{10} & 05 & 1.4348 & 1.8015 & 2.2912 & 2.0248 & 1.9560 & 1.8817 & 1.8134 \\
\hline & 08 & 1.2942 & 2.0632 & 2.7892 & 2.3910 & 2.1912 & 2.0034 & 1.8484 \\
\hline & 10 & 1.2045 & 2.2230 & 3.1413 & 2.6236 & 2.3106 & 2.0403 & 1.8522 \\
\hline \multicolumn{9}{|c|}{$a=0.50$} \\
\hline \multirow{3}{*}{05} & 05 & 1.4636 & 2.0746 & 2.6975 & 2.3628 & 2.2485 & 2.1315 & 2.0275 \\
\hline & 08 & 1.3328 & 2.4461 & 3.4308 & 2.9207 & 2.6349 & 2.3720 & 2.1589 \\
\hline & 10 & 1.2658 & 2.6469 & 3.8845 & 3.2426 & 2.8263 & 2.4660 & 2.1912 \\
\hline \multirow{3}{*}{10} & 05 & 1.4204 & 1.7967 & 2.2860 & 2.0193 & 1.9505 & 1.8769 & 1.8096 \\
\hline & 08 & 1.2678 & 2.0493 & 2.7729 & 2.3760 & 2.1790 & 1.9951 & 1.8440 \\
\hline & 10 & 1.1723 & 2.2057 & 3.1228 & 2.6066 & 2.2978 & 2.0330 & 1.8498 \\
\hline
\end{tabular}

\section{Recommendation}

The absolute relative bias (ARB) of $\hat{\sigma}_{S T_{1}}$ first decreases and then increases steadily as $n_{1}$ increases for all considered values of $\delta$ for fixed $\alpha$ and $n_{2}$. The bias is almost negligible near $\delta=100$. The values of ARB of $\hat{\sigma}_{S T_{1}}$ decrease (increase) as $\alpha\left(n_{2}\right)$ increases for all considered values of $\delta$ except $\delta$ near 1.00 .

The bias of $\hat{\sigma}_{S T_{2}}$ is almost negligible for all considered values of $\delta$. The ARB of $\hat{\sigma}_{S T_{2}}$ decreases as $n_{1}$ increases for all $\delta$ when other parametric values are fixed but it increases as $n_{2}$ increases for all $\delta$ (except $\delta$ near 1.00). The opposite trend has been seen when $\alpha(\leq 0.10)$ increases. The decreasing trend also has been seen as ' $a$ ' increases for $\delta$ $\geq 0.80$ when other values are fixed.

\subsection{Under SELF risk criterion}

The estimator $\hat{\sigma}_{S T_{i}} ; i=1,2$ is more efficient than the estimator $Y_{i} ; i=1,2$ respectively in the effective interval $0.60 \leq \delta \leq 1.80$ for all considered values of the parametric space and attains maximum efficiency at the point $\delta=1.00$.

The relative efficiency $R E_{(S)}\left(\hat{\sigma}_{S T_{1}}, Y_{1}\right)$ increases (decreases) as $n_{2}\left(n_{1}\right)$ increases for $\delta \geq 0.80$ when other values are fixed. In addition, the gain in efficiency decreases with increase of $\alpha$ when $\delta \geq 0.80$ for all considered values of the parametric space.

The relative efficiency $R E_{(S)}\left(\hat{\sigma}_{S T_{2}}, Y_{2}\right)$ increases (decreases) as $n_{2}(\alpha)$ increases for $0.80 \leq \delta \leq 1.6$ (when other parametric values are fixed). The gain in efficiency decreases 
as $n_{1}$ increases. The similar trend has been seen with the increase of ' $a$ ' when $\delta$ is small and other parametric values are fixed.

\subsection{Under ILLF risk criterion}

The estimator $\hat{\sigma}_{S T_{i}} ; i=1,2$ is more efficient than the estimator $Y_{i} ; i=1,2$ respectively in the interval $\delta \geq 0.60$ for all considered values of the parametric space and attains maximum efficiency at the point $\delta=1.00$.

The relative efficiency $R E_{(L)}\left(\hat{\sigma}_{S T_{1}}, Y_{1}\right)$ decreases for $\delta \geq 0.60$ when $n_{1}$ or $\alpha$. In addition, the gain in efficiency increases when $n_{2}$ increases for $\delta \geq 0.80$. It also increases with increase of ' $a$ ' for $\delta \leq 1.00$ when other values are fixed.

The relative efficiency $R E_{(L)}\left(\hat{\sigma}_{S T_{2}}, Y_{2}\right)$ decreases as ' $a$ ' increases for all considered values of $\delta$ when $\alpha>0.05$ and for $\delta \leq 1.00$ when $\alpha \leq 0.05$. Other properties of $\hat{\sigma}_{S T_{2}}$ are similar to $\hat{\sigma}_{S T_{1}}$.

On the basis of relative efficiencies we conclude that the estimator $\hat{\sigma}_{S T_{2}}$ dominates $\hat{\sigma}_{S T_{1}}$ for all considered values of the parametric space except small sample size and $\delta$ near 1.00 when ' $a$ ' is negative i.e., when overestimation is less serious than underestimation. On other hand when overestimation is more serious than underestimation, $\hat{\sigma}_{S T_{2}}$ is preferable over $\hat{\sigma}_{S T_{1}}$ for large $\delta \geq 1.20$.

\section{References}

1. J. L. Folks and R. S. Chhikara, Journal of the Royal Statistical Society, Series-B, 40, 263 (1978).

2. J. L. Folks and R. S. Chhikara, The Inverse Gaussian distribution (Marcel Dekker, New York, 1989).

3. V. Seshadri, The Inverse Gaussian distribution. Statistical Theory and Applications, (SpringerVerlag, New York, 1998).

4. H. R Varian, A Bayesian approach to real estate assessment. In studies in Bayesian econometrics and statistics in honor of L.J. Savage, Eds S.E. Feinberg and A. Zellner (Amsterdam North Holland, 1975) p. 195.

5. A. Zellner, Journal of the American Statistical Association, 81, 446 (1986). doi: $10.2307 / 2289234$

6. J. Rojo, Communication in Statistics - Theory and Methods, 16, 3745 (1987).

7. G. Prakash and D. C. Singh, Austrian Journal of Statistics, 35 (4), 463 (2006).

8. C. P. Han and T. A. Bancroft, Journal of American Statistical Association, 63, 1333 (1968). doi: $10.2307 / 2285888$

9. F. Mosteller, Journal of the American Statistical Association, 43, 231 (1948). doi: $10.2307 / 2280369$

10. T. Kitagawa, University California Publication of Statistics, 3, 147 (1963).

11. B. K. Kale and T. A. Bancroft, Biometrices, 23, 335 (1967). doi:10.2307/2528166

12. J. Singh, Journal of American Statistical Association, 66, 82 (1971). doi: $10.2307 / 2284852$

13. O. Rai, Communication in Statistics - Theory and Methods, 25, 2057 (1996).

14. B. N. Pandey, B. P. Singh and A. K. Srivastava, Aligarh Journal of Statistics, 25, 35 (2005). 